\title{
Peculiarities of hydrodynamics in the evaporation of hydrocarbon droplets
}

\author{
G. Miliauskas, S. Sinkunas, J. Talubinskas \& K. Sinkunas \\ Department of Thermal and Nuclear Energy, \\ Kaunas University of Technology, Lithuania
}

\begin{abstract}
Combustion of liquid fuel generally is going on in the sprayed form. The combustion effectiveness is influenced by the evaporation rate of droplets, which determine heat and mass transfer intensity between droplets and the gas and peculiarities of combined heat spread in warming semitransparent droplets. Modeling of complex heat transfer raises the assessment problem of the potential liquid instability within droplets. Free liquid circulation in warming liquid droplets can occur under the influence of Archimedes forces and forced circulation inside them can be forced by sliding gas friction forces acting on the droplet surface. Under the influence of these forces, droplets liquid starts to flow on its surface and emerged vortices pass into the interior layers of the droplets. Hydrodynamic regime of the liquid droplets determines the solution methodology of the inner "droplet" problem. A combined analytical and numerical method of investigation is applied. A system of parabolic type second order integral-differential equations describing complex heat transfer in the droplet by conduction and radiation is transformed into an infinite series of integral equations. Securing balance of energy fluxes in the droplet with confidence of one hundredth of percent and using the fastest sinking the droplet surface temperature is selected. The change of thermal state and phase transformation intensity of warming hydrocarbons droplets heated in air is modeled under different boundary conditions of heat and mass transfer. The influence of forced liquid circulation on the droplet thermal state evaluated using effective heat conduction coefficient. Free liquid circulation in the droplets is evaluated by Rayleigh number.
\end{abstract}

Keywords: hydrocarbon droplets, combined heat and mass transfer, heating and evaporation of droplets, hydrodynamics. 


\section{Introduction}

Liquid fuel is generally combusted in the sprayed form. Its combustion efficiency determines droplet evaporation speed. Heat and mass transfer processes non-stationarity and their interactions are important factors in the sprinkle liquid fuel heating and evaporation process. High-temperature twophase flow multiple heat and mass transfer processes additionally affect the interaction of translucent droplets absorbed radiation flux. Experimentally investigate the multiple heat transmission of droplets rather difficult, so widely used theoretical methods [1]. Modeling multiple heat spread of the droplets of liquid existence instability assessment problem in its. Spontaneous circulation of liquid warming droplets can occur when Archimedes forces is on, and they forced circulation can lead to gas sliding friction force acting on the surface. The exposure of the liquid droplets flowing on the surface and formed vortices passes into the interior layers of the droplets. Hydrodynamic mode of the liquid droplets determines the solution methodology of "drop" inner problem. The simplest form of droplets heat transfer models that deny the influence of radiation. Suggesting that the droplets while maintaining a uniform temperature is warming up their volume, they concluded "the infinite heat conductivity", or "infinite fluid mixing" strain $[2,3]$. These droplets heat transfer models to define the effective conductivity model [4], which additionally take into account the fluidflow finite intensity of thermal impact on the spread of the droplets. Translucent droplets radiation absorbed by the flow describing the spectral radiation models [5-9] enables the evaluation of the combination of heat spreading droplets. Recently developed combined analytical and numerical models of droplet nonstationary evaporation $[5,10]$. Then the temperature field of droplets of a multiple their heating case describing integral equation the endless lines. Despite their numerical solution schemes, convergence control is convenient, but inconvenience causes the need to know the evaporating droplet surface temperature. When trying to save the counting machine time and avoid this temperature calculation by iterative methods, doing that task simplification assumption [10]. Correctness case the droplet surface temperature is calculating by iterative methods for solving the energy flow balance droplets on the surface of describing an additional equation. In this case, the heat conductivity effective coefficient consider and to hydrodynamic mode impact on the liquid droplets thermal state [5].

The aim of this work is to assess spontaneous fluid flow increases possibility of evaporative and warming pure liquid fuel droplets.

\section{Formulation of the Problem}

Natural circulation of fluid within a droplet is induced by non-uniformity of temperature field, which has been expressed by difference of temperatures at droplet surface and in the center of it: $\Delta T_{l} \equiv T_{R}-T_{C}$. The intensity of natural circulation in the droplet is described by Rayleigh number: 


$$
R a=\frac{\beta_{l} g R^{3}}{v_{l} a_{l}} \Delta T_{l}
$$

The circulation of liquid is said to onset when the Rayleigh number exceeds the critical value i.e. $R a>R a_{c r}$. Otherwise the processes in the droplet are dominated by diffusion. Research of fluid behavior in spherical volume with non-uniform temperature field [11] demonstrates that critical values of the Rayleigh number are strongly dependent on the method of volume heating. For asymmetric heating the critical value is in the range of 200 to 300 , and for symmetric heating it can reach few thousands. The fluid temperature profile in the droplet under heating is described by the following equation [1]:

$$
T_{r}=T_{R}+\frac{2}{r} \sum_{n=1}^{\infty} \sin \frac{n \pi r}{R} \int_{0}^{t} f_{n}(\tau) \exp \left[-a\left(\frac{n \pi}{R}\right)^{2}(t-\tau)\right] d \tau,
$$

where the heat source is described by the function

$$
\begin{aligned}
& f_{n}(\tau)=(-1)^{n} \frac{R}{n \pi} \frac{d T_{R}}{d \tau} \\
& +\frac{1}{\rho c_{p} R} \int_{0}^{R}\left(\sin \frac{n \pi r}{R}-\frac{n \pi r}{R} \cos \frac{n \pi r}{R}\right) q_{r} d r
\end{aligned}
$$

The function takes into account radiant heat absorption in the semitransparent droplet and the dynamics of its surface temperature. The model of the droplet does not consider the slippage between two phases i.e. droplet and its environment. In this scenario the effect of gaseous phase to fluid circulation in the droplet are not considered as the forces inducing such circulation are absent. The droplet is heated by conductivity. The surface temperature of the evaporating droplet is calculated using energy conservation principle and has been implemented using the method of the steepest descent:

$$
\lambda_{v g} \frac{T_{g}-T_{R}}{2 R} f_{B_{T}}-\left.\lambda_{e f} \frac{\partial T_{r}}{\partial r}\right|_{r \equiv R}-m_{v} L \equiv 0 .
$$

Spalding heat transfer parametric function $f_{B_{T}}$ in eq. (4) takes into account Stephan hydrodynamic effect to in-droplet convection of the droplet in the gas stream. It is defined as [4]

$$
f_{B_{T}} \equiv \frac{\ln \left(1+B_{T}\right)}{B_{T}}
$$


where Spalding heat transfer parameter for non-stationary evaporation of droplet is defined as [12]

$$
B_{T} \equiv \frac{c_{p, v g}\left(T_{g}-T_{R}\right)}{L}\left(1-\left.\lambda_{e f} \frac{\partial T}{\partial r}\right|_{r \equiv R} / q_{c, R}\right) .
$$

Non-stationary temperature gradient in eq. (4) and (6) is described by the following equation (2):

$$
\left.\frac{\partial T_{r}}{\partial r}\right|_{r \equiv R}=\frac{2 \pi}{R^{2}} \sum_{n=1}^{\infty} n(-1)^{n} \int_{0}^{t} f_{n}(\tau) \exp \left[-a\left(\frac{n \pi}{R}\right)^{2}(t-\tau)\right] d \tau .
$$

Assuming that there is no forced circulation in the droplet and that the effect of natural circulation to droplet heating is negligible the effective thermal conductivity coefficient in (4) and (6) will be defined by the thermal conductivity coefficient of fluid $\lambda_{e f} \equiv \lambda_{l}$. Vapor mass flux is calculated based on the Shorin-Kuzikovskij model $[13,14]$

$$
m_{v}=\frac{D_{v g}}{T_{R}} \frac{\mu_{g}}{R \cdot R_{\mu}} p \ln \frac{p-p_{v, \infty}}{p-p_{v, R}} .
$$

System of equations (1)-(8) can be solved numerically. Using iterative method number $J$ of the control droplet cross-section is selected arbitrary. The position of the cross-section is defined by the dimensionless droplet coordinate $\eta_{j}\left(\eta_{j}=0\right.$, when $j \equiv 1$ and $\eta_{j}=1$, when $\left.j \equiv J\right)$. Control time $t$ is selected and number $I$ of time coordinate steps is provided ( $\tau=0$, when $i \equiv 1$ and $\tau=t$, when $i \equiv I$ ). Temperature field in the droplet is determined from eq. (2) iteratively varying droplet surface temperature on the basis of energy conservation principle at the surface and using the method of the steepest descent. The final result is achieved when the energy balance error (4) from the previous iteration is no greater than 0.01 percent. The local radiation flux density in a semi-transparent droplet is calculated using the technique described in ref. [5]. It requires the updated temperature field in the droplet from the previous iteration, takes into account light absorption by the droplet and effects at the surface of the droplet.

\section{Results and discussion}

To investigate the influence of fluid natural circulation in the hydrocarbon droplet the droplet heating and evaporation in air flow has been modelled. The nhexane, n-heptane and n-decane have been chosen. The complex droplet heating by conduction and radiation has bean modelled. It is assumed the absolutely black-body radiation heat source with the temperature of the droplet environment exists. The droplet is internally heated by conduction to the temperature of 
equilibrium evaporation $T_{e, k}$, which does not alter during such evaporation mode (fig. 1).
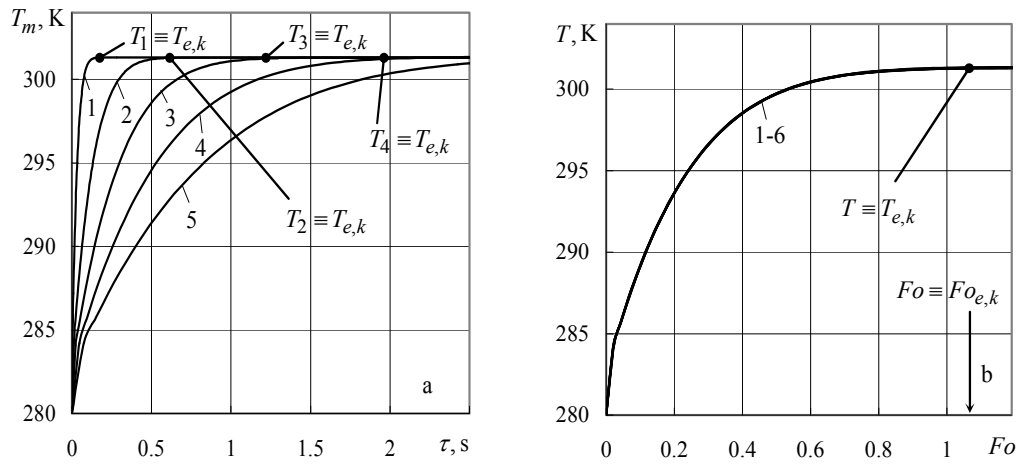

Figure 1: n-hexane droplet internal heating by conduction in air environment. Mean droplet temperature in time (a) and Fourier criterion (b) domains. $R \cdot 10^{6}$, m: $1-50,2-100,3-200,4-300,5-400$, $1-600 ; T_{g}=473 \mathrm{~K}$.

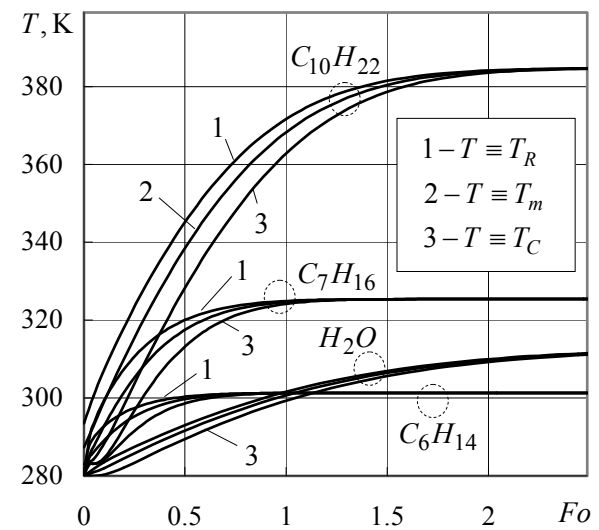

Figure 2: Hydrocarbons n-hexane $\left(\mathrm{C}_{6} \mathrm{H}_{14}\right)$, n-heptane $\left(\mathrm{C}_{7} \mathrm{H}_{16}\right)$, n-decane $\left(\mathrm{C}_{10} \mathrm{H}_{22}\right)$ and water $\left(\mathrm{H}_{2} \mathrm{O}\right)$ droplet heating in $473 \mathrm{~K}$ air temperature.

Dynamics of droplet mean temperature, when internally heating by conduction only, for the same liquid droplets is invariant of droplet size in the domain of Fourier criterion (fig. 1b). For each type of liquid the characteristic curves of droplet surface temperature $T_{R}\left(F_{O}\right)$, center temperature $T_{C}\left(F_{O}\right)$ and mean temperature $T_{m}\left(F_{O}\right)$ dynamics can be established (fig. 2). It allowed optimization of the numerical model for droplet heating and evaporation processes. The characteristic droplet temperature non-uniformity curves are 
established based on the droplet surface temperature and droplet center temperature dynamics. They clearly depict that liquid properties have influence to temperature non-uniformity (fig. 3). The intensive temperature increase of droplet external fluid layers during the initial droplet heating stage causes rapid rise of the droplet temperature non-uniformity. Later the temperature nonuniformity reduces and eventually vanishes when droplet equilibrium evaporation mode is entered. The results show that water droplets establish equilibrium evaporation earlier than hydrocarbon droplets. Therefore, nonuniformity in water droplets vanishes earlier, as well. In the modeled case (fig. 3) non-stationary evaporation of hydrocarbon droplets lasts up to $80 \%$ of phase transformation duration, but for water droplets it is just $20 \%$.

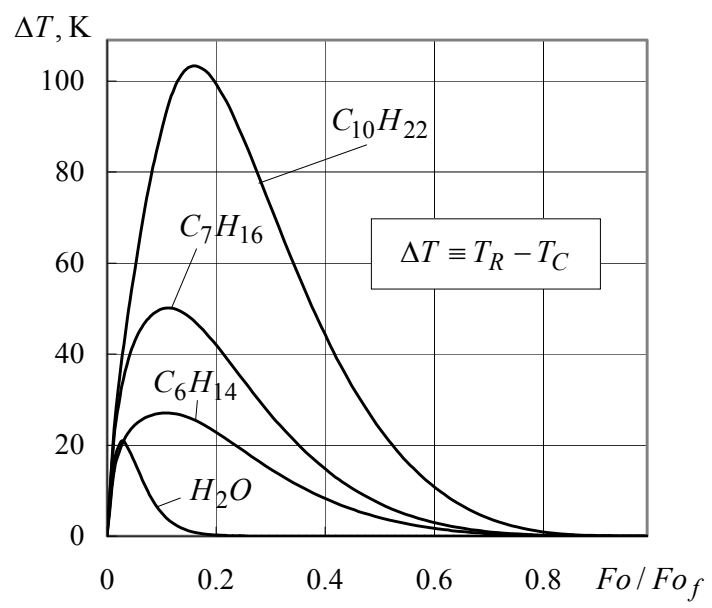

Figure 3: The dynamics of non-uniformity of temperature field for droplets in $1073 \mathrm{~K}$ air environment.

Droplet temperature non-uniformity is significantly influenced by gas phase temperature, which has effect on droplet equilibrium evaporation temperature. For air temperature of $473 \mathrm{~K}$ droplet equilibrium evaporation temperature is $301.3 \mathrm{~K}$ for $\mathrm{n}$-hexane, $325.4 \mathrm{~K}$ for $\mathrm{n}$-heptane, $384.8 \mathrm{~K}$ for $\mathrm{n}$-dekane and $313 \mathrm{~K}$ for water. Droplet equilibrium evaporation temperature increases to $311.3 \mathrm{~K}, 337.8 \mathrm{~K}$, $406.4 \mathrm{~K}$ and $332.1 \mathrm{~K}$ correspondingly when air temperature is increased by $1000 \mathrm{~K}$ to $1473 \mathrm{~K}$. For hydrocarbons with higher saturation temperature (in this instance it was n-dekane) droplet equilibrium evaporation temperature is higher and the spike of temperature non-uniformity is more pronounced (fig. 3). Therefore, conditions for fluid natural circulation are favorable. For sprinkled of liquid temperatures close to droplet equilibrium evaporation temperature droplet temperature non-uniformity will be smaller and will last shorter compared to sprinkled of liquid with temperatures significantly below it.

The research results show and quantify that the size of droplet has an effect to droplet natural circulation (fig. 4). 


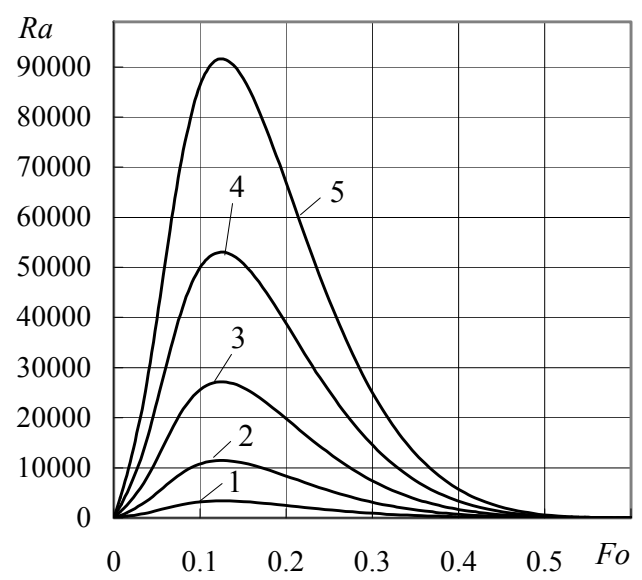

Figure 4: Droplet size influence to droplet internal natural circulation for n-decane. $R \cdot 10^{6}, \mathrm{~m}: 1-500,2-750,3-1000,4-1250,5-1500$; $T_{l, 0}=283 \mathrm{~K}, T_{g}=1073 \mathrm{~K}$.

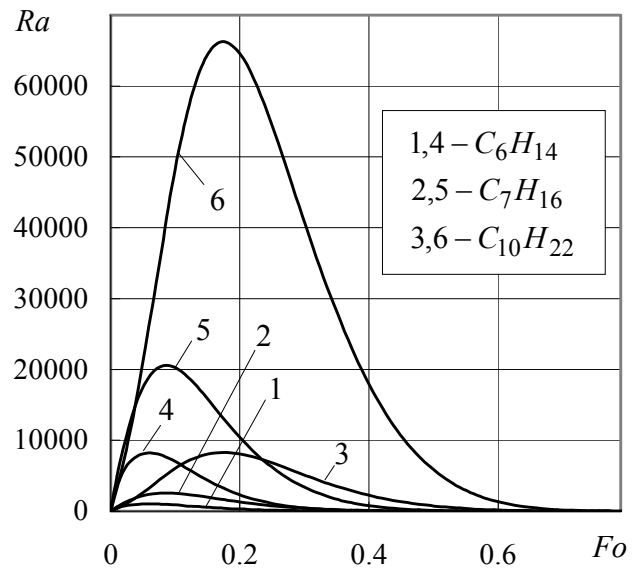

Figure 5: Influence of liquid properties to droplet internal natural fluid circulation. $R \cdot 10^{6}$, m: $1-3-750,4-6-1500 ; T_{l, 0}=283 \mathrm{~K}, T_{g}=873 \mathrm{~K}$.

Larger droplets have better conditions to stimulate droplet natural circulation. The results of this research show that when the size of droplets is the same, the fluid natural circulation will depend on liquid properties. In the case of hydrocarbons, the results show (fig. 5) that out of three hydrocarbons n-dekane (the highest hydrocarbon out of three) had the best properties to stimulate natural circulation. 


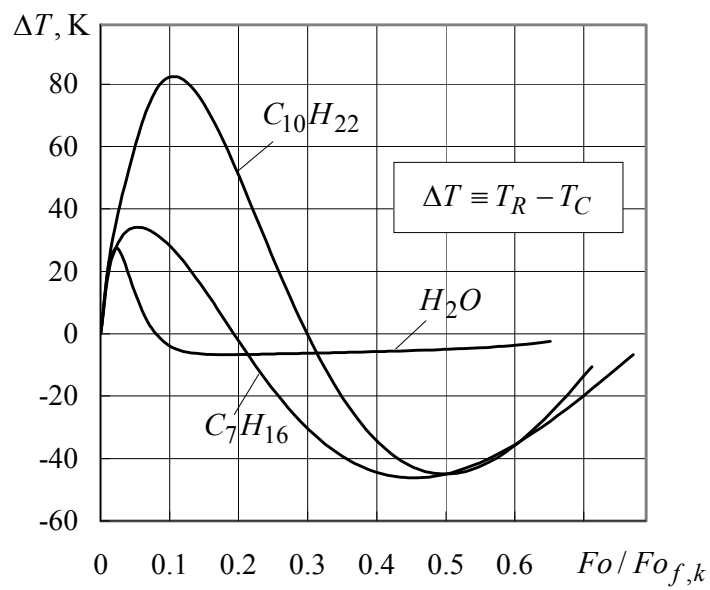

Figure 6: The effect of fluid radiation absorption property to non-uniformity of droplet heating. $R_{0}=500 \cdot 10^{-6} \mathrm{~m}, T_{l, 0}=283 \mathrm{~K}, T_{g}=1073 \mathrm{~K}$.

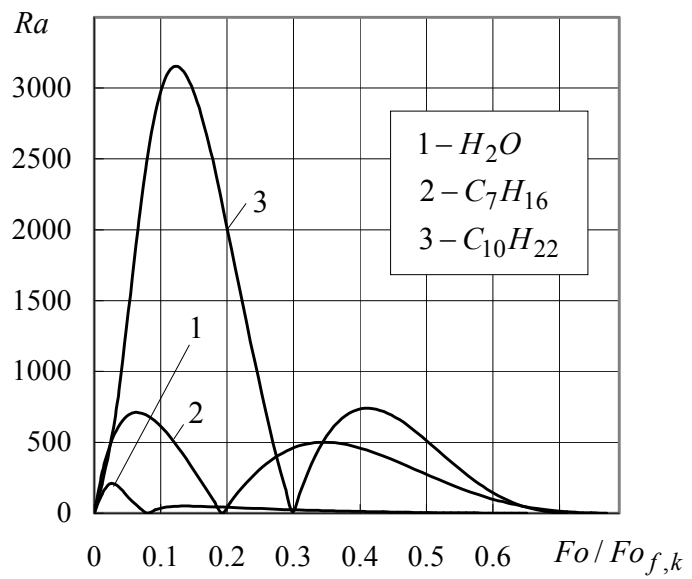

Figure 7: The effect of fluid properties and radiation to droplet internal natural fluid circulation. $R_{0}=500 \cdot 10^{-6} \mathrm{~m}, T_{l, 0}=283 \mathrm{~K}, T_{g}=1073 \mathrm{~K}$.

Absorbed radiation by semitransparent droplet is essential to droplet heating (fig. 6). In the case of complex conduction-radiation droplet heating, the temperature profile in the droplet and the heating process has new features introduced. The process changes quantitatively as well as qualitatively. In the complex heating case two spikes of temperature non-uniformity are observed. The first one is the consequence of conduction and has been discussed above. The second spike is the result of radiation absorption by liquid and results in temperature increase of internal liquid layers. The maximum of this non- 
uniformity spike occurs when droplet equilibrium evaporation is established. The temperature gradient in the droplet balances heat transfer due to radiation and conduction if the fluid internal circulation is insignificant. The second temperature non-uniformity spike is smaller than the first. Therefore, conditions for fluid natural circulation are determined by the first spike (fig. 7).

\section{Conclusions}

On the basis of Rayleigh number utilization to classify water droplets and research results for n-decane, hydrocarbon droplets can be divided into such categories:

- $\quad$ small (less than $100 \mu \mathrm{m}$ in diameter);

- medium $(50-1000 \mu \mathrm{m}$ in diameter);

- $\quad$ large (more than $1 \mathrm{~mm}$ in diameter).

Fluid natural circulation in small hydrocarbon droplets is practically impossible. It is unlikely that natural circulation will be significant in medium size droplets, as well. The only category of hydrocarbon droplets, where fluid natural circulation might be sensible is of large droplets, i.e. with diameters of droplets exceeding $1 \mathrm{~mm}$. In general, the larger the droplet size the more likely that natural circulation in the droplet will be observed.

Radiation absorbed by semitransparent droplets has a significant effect on their heating, evaporation and process dynamics by the formation of the secondary spike in temperature non-uniformity. It is smaller than the first spike originating due to conduction, and does not dominate determination of conditions for fluid natural circulation.

Droplet sizes in technologies utilizing the principle of liquid sprinkle of mostly fall into small and medium categories. Therefore, fluid natural circulation in the droplets is insignificant and is not necessary to be modeled.

\section{Nomenclature}

$a$-thermal diffusivity, $\mathrm{m}^{2} / \mathrm{s} ; B$-Spalding transfer number; $c_{p}$-specific heat, $J /(\mathrm{kg} \mathrm{K}) ; D$ - mass diffusivity, $\mathrm{m}^{2} / \mathrm{s} ; F_{O}$ - Fourier number; $L$-latent heat of evaporation, $\mathrm{J} / \mathrm{kg} ; \mathrm{m}$-vapour mass flux, $\mathrm{kg} /\left(\mathrm{sm}^{2}\right) ; n$-number of the term in infinite sum; $p$-pressure, $P a ; q$-heat flux, $W / \mathrm{m}^{2} ; R$-radius of a droplet, $m$; $r$-coordinate of a droplet, m; $R a$-Rayleigh number; $N u$-Nusselt number; $T$-temperature, $K ; \eta$-non-dimensional coordinate; $\lambda$-thermal conductivity, $W /(m K) ; \mu$-molecular mass, $\mathrm{kg} / \mathrm{kmol} ; \rho$ - density, $\mathrm{kg} / \mathrm{m}^{3} ; \tau$ - time, $\mathrm{s}$;

Subscripts: $C$-droplet centre; $\mathrm{cr}-$ critical; $e$-equilibrium evaporation; $g$-gas; $i$-time index; $I-$ index of control time; $j$-index of droplet crosssection; $J-$ index of droplet surface; $\mathrm{k}$-conductive; $l$-liquid; $m$-mass average; $r$ - coordinate of a droplet; $R$ - droplet surface; $v$-vapour; $v g$ - vapourgas mixture; 0 - initial state; $\infty$ - far from a droplet. 


\section{References}

[1] Sazhin S.S., Advanced models of fuel droplet heating and evaporation. Progress in Energy and Combustion Science, 32, pp 162-214, 2006.

[2] Law C.K., Unsteady droplet combustion with droplet heating. Combust. Flame, 26, pp. 17-22, 1976.

[3] Law C.K., Multicomponent droplet combustion with rapid internal mixing. Combust. Flame, 26, pp. 219-233, 1976.

[4] Abramzon B., Sirignano W.A., Droplet vaporization model for spray combustion calculations. Int. J. Heat Mass Transfer, 32, pp.1605-1618, 1989.

[5] Miliauskas $G$, Regularities of unsteady radiative-conductive heat transfer in evaporating semitransparent liquid droplet. Int. J. Heat Mass Transfer, 44, pp. 785-798, 2001.

[6] Lage P.L.C., Rangel R.H., Single droplet vaporization including thermal radiation absorption. J. of Thermophysics and Heat Transfer, 7, pp. 502509, 1993.

[7] Harpole G.M., Radiative absorption by evaporating droplets. Int. J. Heat Mass Transfer, 22, pp.17-26, 1980.

[8] Dombrovsky L.A., Thermal radiation form no isothermal spherical particles of a semitransparent material. Int. J. Heat Mass Transfer, 43, pp. 1661$1672,2000$.

[9] Tseng, C.C., Viskanta R., Enhancement of water droplet evaporation by radiation absorption. Fire Safety J, 41, pp 236-247, 2006.

[10] Sazhin S, Modeling of heating, evaporation and ignition of fuel droplets: combined analytical, asymptotic and numerical analysis. J. of Physics: Conference Series, 22, pp 174-193, 2005.

[11] Gershuni G.Z., Zhukovickiy E.M., Konvektivnaja ustoicivost neszimaemoi zidkosti, Nauka, Moskva, 1972.

[12] Miliauskas G., Interaction of the transfer processes in semitransparent liquid droplets. Int. J. Heat Mass Transfer, 46, pp. 4119-4138, 2003.

[13] Shorin S.N. Teploperedacha, Vishaja Shkola, Maskva, 1964.

[14] Kuzikovskij A.V. Dynamic of spherical particle in powerful optical field, Izv. VUZ Fizika,. 5, pp 89-94, 1970. 